University of Nebraska - Lincoln

DigitalCommons@University of Nebraska - Lincoln

\title{
Regulatory control of high levels of carotenoid accumulation in potato tubers
}

\author{
Xiangjun Zhou \\ USDA-ARS \\ Ryan McQuinn \\ Cornell University \\ Zhangjun Fei \\ Cornell University \\ Anne-Marie A. Wolters \\ Wageningen University \\ Joyce Van Eck \\ Cornell University
}

See next page for additional authors

Follow this and additional works at: https://digitalcommons.unl.edu/usdaarsfacpub

Part of the Agricultural Science Commons

Zhou, Xiangjun; McQuinn, Ryan; Fei, Zhangjun; Wolters, Anne-Marie A.; Van Eck, Joyce; Brown, Charles; Giovannoni, James J.; and $\mathrm{Li}$, $\mathrm{Li}$, "Regulatory control of high levels of carotenoid accumulation in potato tubers" (2011). Publications from USDA-ARS / UNL Faculty. 611.

https://digitalcommons.unl.edu/usdaarsfacpub/611

This Article is brought to you for free and open access by the U.S. Department of Agriculture: Agricultural Research Service, Lincoln, Nebraska at DigitalCommons@University of Nebraska - Lincoln. It has been accepted for inclusion in Publications from USDA-ARS / UNL Faculty by an authorized administrator of DigitalCommons@University of Nebraska - Lincoln. 


\section{Authors}

Xiangjun Zhou, Ryan McQuinn, Zhangjun Fei, Anne-Marie A. Wolters, Joyce Van Eck, Charles Brown, James J. Giovannoni, and Li Li 


\title{
Regulatory control of high levels of carotenoid accumulation in potato tubers
}

\author{
XIANGJUN ZHOU ${ }^{1,2}$, RYAN MCQUINN ${ }^{1,3}$, ZHANGJUN FEI $^{1,3}$, ANNE-MARIE A. WOLTERS ${ }^{4}$, JOYCE VAN ECK ${ }^{3}$, \\ CHARLES BROWN 5 , JAMES J. GIOVANNONI ${ }^{1,3} \&$ LI LI $^{1,2}$ \\ ${ }^{1}$ Robert W. Holley Center for Agriculture and Health, USDA-ARS, ${ }^{2}$ Department of Plant Breeding and Genetics, ${ }^{3}$ Boyce \\ Thompson Institute for Plant Research, Cornell University, Ithaca, NY 14853, USA, ${ }^{4}$ Laboratory of Plant Breeding, \\ Wageningen University, PO Box 386, 6700 AJ Wageningen, the Netherlands and ${ }^{5}$ USDA-ARS, 24106 N. Bunn Road., Prosser, \\ WA 99350, USA
}

\begin{abstract}
Potato (Solanum tuberosum L.) tubers contain a wide range of carotenoid contents. To decipher the key factors controlling carotenoid levels in tubers, four potato lines (Atlantic, Désirée, 91E22 and POR03) were examined by a combination of biochemical, molecular and genomics approaches. These lines contained incremental levels of carotenoids, which were found to be associated with enhanced capacity of carotenoid biosynthesis as evident from norflurazon treatment. Microarray analysis of high and low carotenoid lines (POR03 versus Atlantic) revealed 381 genes that showed significantly differential expression. The carotenoid metabolic pathway genes $\beta$-carotene hydroxylase $2(\mathrm{BCH} 2)$ and $\beta$-carotene hydroxylase 1 (BCH1), along with zeaxanthin epoxidase (ZEP), and carotenoid cleavage dioxygenase $1 A$ (CCD1A) were among the most highly differentially expressed genes. The transcript levels of $\mathrm{BCH} 2$ and $\mathrm{BCH} 1$ were lowest in Atlantic and highest in POR03, whereas those of $\mathrm{ZEP}$ and $C C D 1 A$ were high in low carotenoid lines and low in high carotenoid lines. The high expression of $\mathrm{BCH} 2$ in POR03 line was associated with enhanced response to sugars. Our results indicate that high levels of carotenoid accumulation in potato tubers were due to an increased metabolic flux into carotenoid biosynthetic pathway, as well as the differential expression of carotenoid metabolic genes.
\end{abstract}

Key-words: $\mathrm{BCH} 2$ promoter; carotenoids; microarray; potato; sugar response.

\section{INTRODUCTION}

Carotenoids are indispensable for human nutrition and health. They provide precursors of vitamin A and offer protection against certain chronic diseases, such as cancer, heart diseases and age-related eye diseases (Fraser \& Bramley 2004). Humans cannot make carotenoids from endogenous precursors and only obtain them from diet. Therefore, development of carotenoid-enriched food crops

Correspondence: L. Li. Fax: +1-607-2551132; e-mail: ll37@ cornell.edu

(C) 2011 Blackwell Publishing Ltd will maximize the nutritional and health benefits of carotenoids to humans. A comprehensive understanding of the regulation of carotenoid accumulation is critical in providing novel strategies for such endeavour.

Carotenoids accumulate in many flowers, fruits and vegetables, giving yellow, orange and red colours. Carotenoid accumulation in these tissues and organs is the net result of biosynthesis, degradation and stable storage of synthesized products (Lu \& Li 2008; Cazzonelli \& Pogson 2010). Multiple factors control the broad diversity of carotenoid composition and content in storage tissues. Regulation of the catalytic activity of carotenoid biosynthesis (Fig. 1) is generally recognized to be important in controlling the final levels of carotenoid accumulation. Phytoene synthase (PSY) is the rate-limiting step in the carotenoid biosynthetic pathway and manipulation of PSY expression in many plants has been demonstrated to enhance carotenoid synthesis by directing metabolic flux into the carotenoid biosynthetic pathway (Shewmaker et al. 1999; Fraser et al. 2002; Ducreux et al. 2005). Transcriptional regulation of carotenogenic gene expression has been shown to be important in controlling specific carotenoid accumulation in some cases (Ronen et al. 1999; Harjes et al. 2008) but not in others (Li et al.2001). A family of carotenoid cleavage dioxygenases (CCDs) catabolizes enzymatic degradation of carotenoids. Expression of these genes inversely regulates carotenoid accumulation (Kato et al. 2006; Ohmiya et al. 2006; Campbell et al.2010). In addition, regulation of stable storage in organelles represents another level of control. Induction of chromoplast formation and increase of chromoplast compartment size and number also impose a strong effect on final carotenoid levels in storage tissues (Liu et al. 2004; Lu et al. 2006; Galpaz et al. 2008).

The growing interest in having foods enriched with carotenoids have prompted the recent and significant progress in breeding and metabolic engineering of carotenoids in crops (Davuluri et al. 2005; Taylor \& Ramsay 2005; Giuliano et al. 2008; Naqvi et al. 2010; Yan et al. 2010). Potato as the world's fourth largest crop generally contains low levels of tuber carotenoids, especially $\beta$-carotene, the most potent provitamin A carotenoid. The main carotenoids present in cultivated potato tubers include lutein, zeaxanthin and 


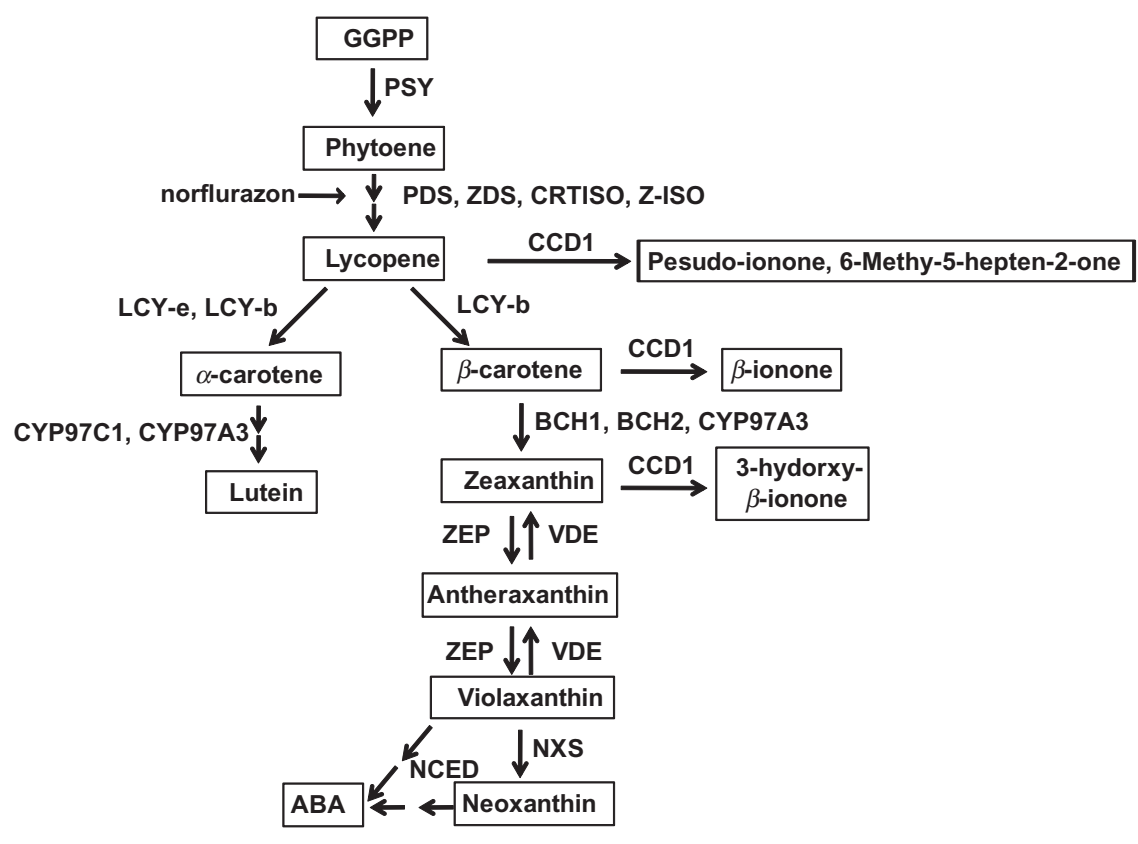

Figure 1. Carotenoid biosynthesis pathway in plants. PSY, phytoene synthase; PDS, phytoene desaturase; ZDS, $\zeta$-carotene desaturase; CRTISO, carotenoids isomerase; Z-ISO, 15-cis- $\zeta$-carotene isomerase; LCY-e, lycopene $\varepsilon$-cyclase; LCY-b, lycopene $\beta$-cyclase; $\mathrm{BCH} 1, \beta$-carotene hydroxylase $1 ; \mathrm{BCH} 2, \beta$-carotene hydroxylase 2 ; CYP97C1, cytochrome P450-type carotene $\varepsilon$-hydroxylase; CYP97A3, cytochrome P450-type monooxygenase; ZEP, zeaxanthin epoxidase; VDE, violaxanthin deepoxidase; NXS, neoxanthin synthase; NCED, 9-cis-epoxycarotenoid dioxygenase; CCD1, carotenoid cleavage dioxygenase 1. Norflurazon inhibition site is indicated. violaxanthin (Burgos et al. 2009). Over the past years, a large effort has taken place to alter carotenoid content and composition in this important food crop. 'Golden' potato tubers with enhanced $\beta$-carotene content have been generated through expression of a carotenoid mini-pathway, including PSY, a bacterial phyotene desaturase (CrtI) and lyopene $\beta$-cyclase (LYC-b) (Diretto et al. 2007, 2010). Tuber-specific overexpression of PSY leads to increased $\beta$-carotene and total carotenoid levels (Ducreux et al.2005). Conversely, silencing of $\beta$-carotene hydroxylase $(\mathrm{BCH})$, zeaxanthin epoxidase $(Z E P)$ and $C C D 4$ results in increased $\beta$-carotene, zeaxanthin or violaxanthin content in tubers (Romer et al. 2002; Van Eck et al. 2007; Campbell et al. 2010). Enhancement of sink strength by expression of the cauliflower $\mathrm{Or}$ mutant gene increases $\beta$-carotene and total carotenoids in tubers (Lu et al. 2006; Lopez et al. 2008). Moreover, potato varieties with high carotenoid levels have been generated through traditional breeding (Brown et al. 2008; Kobayashi et al. 2008).

Genetic studies of the inheritance of carotenoids in potato tubers have identified the $Y$ locus in chromosome 3 as the major regulatory allele, controlling yellow and white flesh colour of tubers (Bonierbale, Plaisted \& Tanksley 1988). The $Y$ gene has been associated with $B C H$ and additional modifying genes are suggested to be involved in controlling total carotenoid levels (Brown et al. 2006). Indeed, $B C H$ was found to be responsible for a major quantitative trait locus for flesh colour (Kloosterman et al. 2010). By association analysis of potato natural diversity, the yellowfleshed tubers which accumulate high levels of zeaxanthin were found to be controlled by a combination of the dominant $\mathrm{BCH} 2$ allele and homozygous recessive $\mathrm{ZEP}$ allele (Wolters et al. 2010). A recent study also reveals the association of low expression of CCD4 with carotenoid content in yellow-fleshed tubers (Campbell et al. 2010).
To gain a better understanding of the control of carotenoid accumulation in potato tubers, we examined carotenogenesis in four potato cultivars (Atlantic, Désirée, 91E22 and POR03) that contain undetectable to high levels of carotenoids. Genome-wide identification of genes responsible for carotenoid accumulation was conducted by comparative microarray analysis of the expression profiles of Atlantic (negligible amount of carotenoids) and POR03 (high carotenoids) tubers. A number of genes associated with carotenoid metabolism were identified from approximately 12000 spots and $\mathrm{BCH} 2$ was found to be the most highly differentially expressed gene. To dissect the regulatory control of carotenoid biosynthetic gene expression in tubers, we isolated the promoters of $\mathrm{BCH} 2$ from potato lines and examined the effect of sugars in activating $\mathrm{BCH} 2$ expression.

\section{MATERIALS AND METHODS}

\section{Plant materials}

Potato tetraploids (Solanum tuberosum L.) Atlantic and Désirée, as well as diploids 91E22 (Brown et al. 2006) and POR03 were used in this study. POR03 (POR03PG6-3) was a selection from the cross EGA9403-4×91E22. EGA9403-4 was a diploid selection from true seeds obtained from the Polish Parental Line Breeding Department, IHAR, in Mlochow, Poland. These two tetraploid varieties and two diploid lines were selected, as most cultivated varieties are tetraploids with low carotenoids, and high carotenoid lines have only been observed among diploid lines. Moreover, a recent study shows that the ploidy change between diploid and tetraploid genotypes causes no statistically significant gene expression difference in the potato genome (Stupar et al. 2007). These potato lines were 
grown in a greenhouse with a $14 \mathrm{~h}$ light/10 h dark photoperiod at $24{ }^{\circ} \mathrm{C}$ for over 3 months. Mature, freshly harvested tubers were used immediately for norflurazon (a phytoene desaturase inhibitor) inhibition studies or frozen in liquid nitrogen and stored at $-80{ }^{\circ} \mathrm{C}$ for RNA extraction and highperformance liquid chromatography (HPLC) analysis.

\section{Norflurazon treatment of potato tubers}

Freshly harvested potato tubers were sterilized with $70 \%$ ethanol for $2 \mathrm{~min}$ and then $30 \%$ bleach for $15 \mathrm{~min}$, followed by rinsing with sterile water for five times. After skins were removed, tubers were cut into thin slices and placed on the BCS medium (Li et al. 2006) containing $0.1 \mathrm{mM}$ norflurazon. The treated tubers were maintained at $24{ }^{\circ} \mathrm{C}$ with a $16 \mathrm{~h} \mathrm{light} / 8 \mathrm{~h}$ dark photoperiod for up to $14 \mathrm{~d}$ in an incubator. Tuber tissue was harvested at $0,3,7$ and $14 \mathrm{~d}$ posttreatment and stored at $-80{ }^{\circ} \mathrm{C}$. The experiments were repeated twice.

\section{HPLC analysis of carotenoids in potato tubers}

Carotenoids were extracted from potato tubers samples as described previously (Lopez et al.2008), and analysed using a Waters HPLC system with a Spherisorb ODS2 $(5 \mu \mathrm{m}$ particle size) reversed-phase $\mathrm{C} 18$ column and a photodiode array detector (Waters, Milford, MA, USA). Individual carotenoids were identified and quantified as described previously (Li et al. 2001).

\section{RNA extraction and microarray hybridization}

At least five tubers from each potato line were cut into small pieces and pooled. Four grams of tuber samples from each line was used for RNA extraction. Total RNA was extracted by a hexadecyltrimethylammonium bromide (CTAB)/phenol method (Griffiths et al. 1999) and treated with RQ1 DNase (Promega, Madison, WI, USA) to completely remove genomic DNA. Fifty micrograms of total RNA was used to generate cDNA and labelled using the 3DNA Array Detection Array 50 Kit (Genisphere, Hatfield, PA, USA). Cy3 or Cy5-labelled cDNA probes were hybridized onto the tomato TOM2 long oligo arrays available through the Boyce Thompson Institute for Plant Research (http://ted.bti.cornell.edu/cgi-bin/TFGD/order/ home.cgi). Microarray hybridization and data acquisition were carried out as reported previously (Moore et al. 2005). The hybridization was performed with four biological replicates.

\section{Microarray data analysis}

Spots with mean signal intensities less than local background intensities plus two standard deviations of the local background in both channels were regarded as empty spots and not included in the downstream statistical analysis. A print-tip Lowess normalization strategy was applied to normalize the ratio values for each array using the marray package (Yang et al. 2002) in Bioconductor (http:// www.bioconductor.org/). Significances of gene expression changes between Atlantic and POR03 tubers were identified using the Patterns from Gene Expression package (PaGE) (Grant, Liu \& Stoeckert 2005). Genes with a false discovery rate (FDR) (Benjamini \& Hochberg 1995) less than 0.1 and a fold change not less than 1.5 were considered as differentially expressed genes. In addition, genes with fold changes no less than 1.5 in all four individual biological replicates were also considered as differentially expressed genes. Functional classification of differentially expressed genes was performed using the Plant MetGenMAP system (Joung et al. 2009). The entire array data set and the experimental descriptions were deposited into the Tomato Functional Genomics Database (Fei et al. 2011).

\section{RNA gel blot and quantitative RT-PCR (qRT-PCR)}

For Northern blot analysis, total RNA $(20 \mu \mathrm{g})$ from potato tubers were resolved on $1.2 \%$ denaturing gels containing formaldehyde and blotted onto Hybond-N + membrane (GE Healthcare, Piscataway, NJ, USA) following the method as described previously (Li et al. 2001). Filters were probed overnight with $\mathrm{P}^{32}$-labelled target gene fragments in ULTRAhyb hybridization buffer (Ambion, Austin, TX, USA). The templates were amplified from potato cDNA using gene-specific primers listed in Supporting Information Table S1, and labelled with random primers ( $\mathrm{Li}$ et al. 2001). Following hybridization, filters were washed twice with $2 \times$ saline-sodium citrate (SSC), $0.1 \%$ sodium dodecyl sulfate (SDS) and $0.2 \times \mathrm{SSC}, 0.1 \% \mathrm{SDS}$ at $42{ }^{\circ} \mathrm{C}$, and then detected using STORM 860 PhosphorImager (GMI, Ramsey, MN, USA).

For qRT-PCR, the cDNA was synthesized using Superscript reverse transcriptase (Invitrogen, Carlsbad, CA, USA). qRT-PCR was conducted by using SYBR Green PCR master mix (Applied Biosystems, Foster City, CA, USA). The cycling conditions involved denaturation at $95^{\circ} \mathrm{C}$ for $10 \mathrm{~min}$ followed by 40 cycles of $95^{\circ} \mathrm{C}$ for $15 \mathrm{~s}$ and $60{ }^{\circ} \mathrm{C}$ for $60 \mathrm{~s}$. The specificity of qRT-PCR was examined by the dissociation curve. The relative expression of ZEP and CCD1A was normalized to a potato actin gene (GenBank accession number GQ339765). Genespecific primers used are listed in Supporting Information Table S1.

\section{Determination of $\mathrm{BCH} 2$ and $\mathrm{ZEP}$ allele composition}

BCH2 allele composition of diploids 91E22 and POR03 and tetraploids Atlantic and Désirée was determined by sequencing PCR products obtained with primers CHY2ex4F and Beta-R822 (Wolters et al. 2010). ZEP allele composition was determined by sequencing PCR products obtained with primers AWZEP9 and AWZEP10 (Wolters et al. 2010). 


\section{Isolation of promoters of $\mathrm{BCH} 2$ genes from potato}

The promoter of $\mathrm{BCH} 2$ from POR03 was isolated using GenomeWalker Universal Kit (Clontech, Mountain View, CA, USA). Based on the sequences of potato BCH2/crtR-b2 cDNA (GenBank accession number: GU233534) and tomato $c r t R$ - $b 2$ cDNA (GenBank accession number: AK246159), two gene-specific primers B2R1and B2R2 were designed to amplify the promoter sequence. Genomic DNA was extracted from potato leaf tissues as described previously (Lopez et al. 2008). DNA $(2.5 \mu \mathrm{g})$ was digested separately with DraI, EcoRI, PvuII and SwaI, and then ligated to GenomeWalker adaptors to create the libraries. The libraries were then used as templates in the nested PCR with adaptor primer 1 and $\mathrm{B} 2 \mathrm{R} 1$ as primers in the first round of amplification, and nested adaptor primer 2 and B2R2 as primers in the second round of amplification. PCR products were cloned into pCR2.1 vector (Invitrogen) and sequenced. Based on the promoter sequence obtained from POR03, a new pair of primers NBPF1 and NBPR1 was designed to amplify the corresponding promoter regions from other varieties. The PCR products were cloned and verified by sequencing.

The promoter sequence from the $B C H 2$ allele 1 was determined by sequencing PCR products obtained with primer combinations NBPF2 + NBPR2 and UBPF1+ NBPR4, using DNA from monoploid genotype 7322 (Wolters et al. 2010) as template. The promoter sequence from $\mathrm{BCH} 2$ allele 2 was determined by sequencing PCR products obtained with primer combinations NBPF2 + NBPR3 and UBPF1 + NBPR4, using DNA from monoploid genotype M5 (Wolters et al. 2010) as template. The search for cis-elements in the promoters was performed by PLACE (Higo et al. 1999).

\section{Construction of PrO $_{\mathrm{BCH} 2}$ :GUS ( $\beta$-glucuronidase) plasmids and Arabidopsis transformation}

To create the $\mathrm{PrO}_{\mathrm{BCH}}$ :GUS constructs, approximately $1.5 \mathrm{~kb}$ promoter sequence from Atlantic (allele 5) and POR03 (allele 3) were cloned into the pSG506 vector and then the chimeric genes were subcloned into pCAMBIA1300 vector as described previously (Chiu et al. 2010). The resulting PrO $_{B C H 2-5}$ : GUS and PrO $_{B C H 2-3}$ :GUS constructs were introduced into Agrobacterium tumefaciens GV3101 and transformed into Arabidopsis thaliana (ecotype Columbia) by the floral dip method.

\section{Sugar treatment of Arabidopsis leaves}

Four-week-old wild-type and $\mathrm{PrO}_{\mathrm{BCH}}$ : GUS transgenic Arabidopsis rosette leaves were detached and placed into $\mathrm{H}_{2} \mathrm{O}$, $6 \%$ glucose and 6\% sucrose, respectively, and shaken for $24 \mathrm{~h}$ at room temperature in the dark (Zhou, Cooke \& $\mathrm{Li}$ 2010). The treated rosette leaves were harvested and frozen immediately in liquid nitrogen.

\section{Sugar determination in potato tubes}

Dried potato tubers $(0.02 \mathrm{~g})$ were homogenized and extracted with boiling water for $15 \mathrm{~min}$. After centrifugation at $13000 \mathrm{~g}$ for $5 \mathrm{~min}$, the supernatants were diluted 20-fold, and analysed by HPLC using a CarboPac PA1 $4 \times 250 \mathrm{~mm}$ analytical column and a CarboPac PA1 $4 \times 50 \mathrm{~mm}$ guard column (Dionex, Vienna, Austria) as described (Kaplan \& Guy 2004).

\section{Determination of GUS activity}

Quantitative analysis of GUS activity was measured with 4-methylumbelliferyl-D-glucuronide (4-MUG) as substrate as described previously (Chiu et al. 2010). Proteins were extracted from 4-week-old Arabidopsis rosette leaves in GUS extraction buffer (50 mM NaHPO 4 pH 7.0, $10 \mathrm{mM}$ 2-mercaptoethanol, $10 \mathrm{mM} \mathrm{Na}{ }_{2}$ EDTA, $0.1 \%$ sodium lauryl sarcosine, $0.1 \%$ Triton $\mathrm{X}-100$ ) and their concentrations were determined by Bradford method (Bio-Rad, Hercules, CA, USA). Protein extracts $(20 \mu \mathrm{L})$ were mixed with $180 \mu \mathrm{L} 1 \mathrm{mM} 4-\mathrm{MUG}$ in extraction buffer, and incubated at $37{ }^{\circ} \mathrm{C}$ for $1 \mathrm{~h}$. A $20 \mu \mathrm{L}$ aliquot was transferred into $80 \mu \mathrm{L}$ $0.2 \mathrm{M} \mathrm{Na}_{2} \mathrm{CO}_{3}$ and fluorescence was measured on a Dynex Fluorite 1000 fluorescence plate reader (Dynex Technologies, Chantilly, VA, USA) with excitation at $365 \mathrm{~nm}$ and emission at $450 \mathrm{~nm}$. The standard curve was made by measuring 4-MU fluorescence. Four independent Pro $_{B C H 2}: G U S$ lines from each construct were used in the assay and the analysis was repeated three times.

\section{RESULTS}

\section{Carotenoid accumulation in potato cultivars}

Four potato lines (Atlantic, Désirée, 91E22 and POR03) were chosen to examine the regulation of carotenoid accumulation in tubers. Among them, Désirée had pink or red skin, and the rest yellow or light-brown skins. The four potato lines displayed different flesh colours. Atlantic was white, Désirée was light yellow and 91E22 and POR03 were orange-yellow and deep orange-yellow, respectively. HPLC analysis revealed that the four cultivars contained different levels of carotenoids. Atlantic contained undetectable amount of carotenoids, whereas POR03 had the highest amount of total carotenoids (Table 1). The increased levels of carotenoid accumulation in these four lines are consistent with the change of flesh colour from white to deep orange-yellow, indicating that carotenoids contribute significantly to tuber flesh colour.

The major carotenoids present in potato tubers are lutein, zeaxanthin and violaxanthin (Burgos et al. 2009). The composition of carotenoids varied among Désirée, 91E22 and POR03. The composition and relative levels of individual carotenoids are shown in Table 1. Lutein was the main carotenoid found in Désirée as shown in previous studies (Morris et al. 2004; Lopez et al. 2008). Zeaxanthin was the predominant carotenoid in 91E22 and POR03. 


\begin{tabular}{lllll}
\hline & Violaxanthin* & Lutein* & Zeaxanthin* & Total* \\
\hline Atlantic & n.d. & n.d. & n.d. & n.d. \\
Désirée & n.d. & $0.43 \pm 0.05$ & n.d. & $0.43 \pm 0.05$ \\
91E22 & $0.15 \pm 0.05$ & $0.22 \pm 0.02$ & $4.85 \pm 0.30$ & $5.21 \pm 0.37$ \\
POR03 & $0.55 \pm 0.51$ & $0.45 \pm 0.25$ & $9.13 \pm 2.13$ & $10.14 \pm 2.90$ \\
\hline
\end{tabular}

Table 1. Major carotenoids in freshly-harvested potato tubers

*Carotenoid levels were expressed as $\mu \mathrm{g} \mathrm{g}^{-1}$ fresh weight. n.d., not detectable.

\section{Increased carotenoid accumulation was associated with enhanced metabolic flux in potato tubers}

Higher accumulation of carotenoids in plants could result from enhanced carotenogenic activity, reduced degradation and/or increased sink strength (Li et al. 2006; Li \& Van Eck 2007). To decipher the mechanisms underlying carotenoid accumulation in potato cultivars, we compared the metabolic flux changes among the four cultivars following norflurazon treatment of the tubers. Norflurazon is known to be a pyridazinone herbicide, which specifically inhibits phytoene desaturase in the carotenoid biosynthetic pathway, resulting in the accumulation of phytoene (Breitenbach, Zhu \& Sandmann 2001). The level of phytoene accumulation reflects the capacity of carotenoid biosynthesis in the tissue (Li et al. 2006). In all cultivars, phytoene was hardly detected in the freshly harvested tubers. In the presence of norflurazon, the phytoene level showed a general continuous increase during the $14 \mathrm{~d}$ of treatment, while the contents of the other carotenoids, i.e. lutein, violaxanthin and/or zeaxanthin, in these potato lines either remain constant or decrease. POR03, 91E22, Désirée and Atlantic accumulated phytoene at decreasing abundance, with the first three varieties containing about 7.4-, 4.2- and twofold more phytoene, respectively, than Atlantic at $7 \mathrm{~d}$ of treatment (Fig. 2). The results indicate that the increased levels of carotenoid accumulation in these four lines were associated with increased capacity of carotenoid biosynthesis.

\section{Identification of differentially expressed genes by microarray analysis}

To further explore the molecule basis of carotenoid accumulation in potato tubers, microarray analysis was employed to examine differences in gene expression between the carotenoid-undetectable cultivar Atlantic and the carotenoid-rich line POR03. Tomato and potato share significant gene sequence identities (Bonierbale et al.1988), and tomato cDNA chips have been used for identification of differentially expressed genes from other related plant species (Ponce-Valadez et al. 2009). Thus, tomato TOM2 long oligonucleotide microarray slides were used to hybridize with Cy3- and Cy5-labelled cDNAs generated from total RNA of Atlantic and POR03 tubers. Approximately 12120 spots were detected, among which 208 genes (1.72\%) showed significantly higher expression (Supporting Information Table S2) and $173(1.43 \%)$ showed significantly lower expression in POR03 than Atlantic (Supporting Information Table S3). These genes were involved in various processes, including metabolic process, response to stresses, biosynthetic and cellular process (Fig. 3). The major group of genes functions in metabolic process, suggesting that complex metabolic changes occur in POR03 tubers. Four carotenoid metabolic pathway genes were identified to be among the differentially expressed genes between POR03 and Atlantic. Among the highly up-regulated genes, BCH2 (Y14810) and BCH1 (SGNU233360) exhibited 21.5- and 8.2-fold increase, respectively, in POR03 over Atlantic. Among the down-regulated genes in POR03, the expression of ZEP (Z83835) and carotenoid cleavage dioxygenase 1A (CCD1A; AY576001) (Simkin et al. 2004) in POR03 was markedly reduced, with 3.6- and 2.1-fold decrease, respectively, when compared to their expression in Atlantic.

\section{Expression of the differentially expressed carotenoid genes was correlated with carotenoid levels in the potato lines}

To validate the microarray results and to find out whether the expression of the four differentially expressed

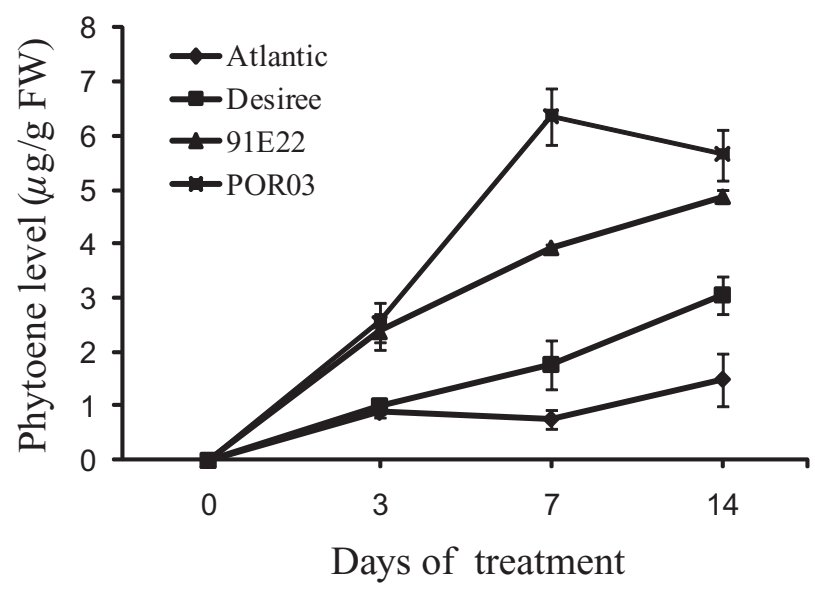

Figure 2. Phytoene synthesis in freshly harvested tuber slices treated with norflurazon. Fresh potato tubers were sterilized and tuber slices were placed on BCS medium containing $0.1 \mathrm{mM}$ norflurazon for up to $14 \mathrm{~d}$. Tuber slices were collected at the indicated time points. Phytoene levels were measured by high-performance liquid chromatography. Error bars indicate $\pm \mathrm{SD}(n=4)$. 


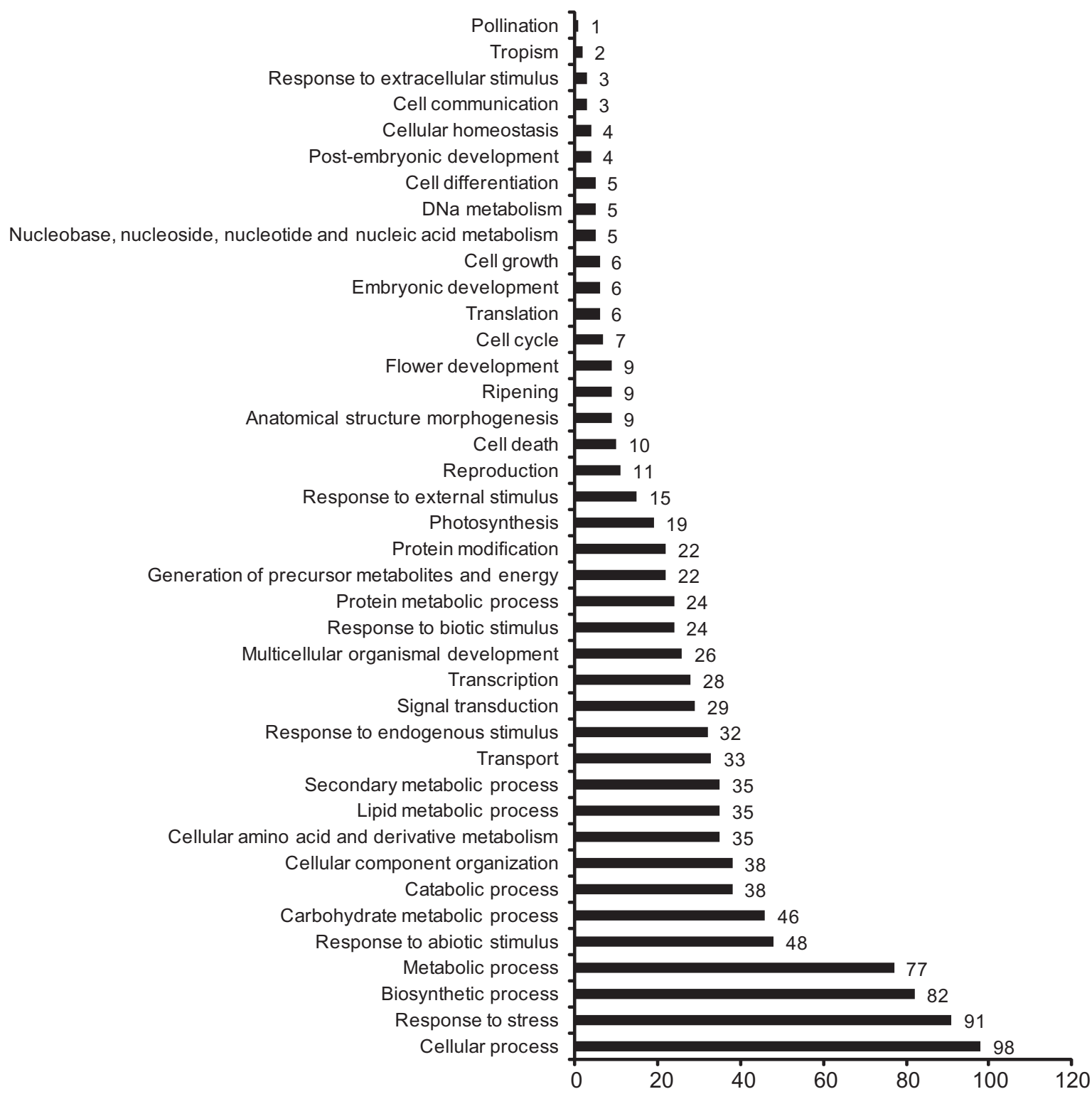

Figure 3. Functional categories of genes differentially expressed in POR03 and Atlantic tubers.

carotenoid genes was correlated with carotenoid content in the tubers, the transcript levels of $\mathrm{BCH} 2, \mathrm{BCH1}, \mathrm{ZEP}$ and $C C D 1 A$ were examined by RNA gel blot or qRT-PCR analysis in the four potato lines. We observed that $\mathrm{BCH} 2$ was expressed higher in carotenoid-rich lines POR03 and 91E22 than in low carotenoid cultivars Désirée and Atlantic, implicating that the enhanced expression of potato $\mathrm{BCH} 2$ could account for higher accumulation of carotenoids in these potato varieties. In addition, $B C H 1$ showed weak expression in these four lines, but it clearly was expressed higher in POR03 than the other varieties (Fig. 4a). Expression of $Z E P$ and $C C D 1 A$ was analysed by qRT-PCR. The results showed that the relative expression levels of $Z E P$ and $C C D 1 A$ was higher in low carotenoidcontaining cultivars (Atlantic and Désirée) and lower in carotenoid-rich cultivars (91E22 and POR03), showing a negative association with carotenoid accumulation in potato tubers (Fig. 4b).

\section{$B C H 2$ and ZEP allele composition}

In the diploid genotypes 91E22 and POR03, each gene may contain two different alleles, while in the tetraploid genotypes Atlantic and Désirée, up to four different alleles for each gene may be present. The alleles for $\mathrm{BCH} 2$ and $Z E P$ are distinguished by specific single-nucleotide polymorphisms (SNPs) as shown in Supporting Information Tables S1 and S3 from a previous publication (Wolters et al. 2010). The allelic composition for $B C H 2$ and $Z E P$ genes in these four potato lines was determined by amplification and sequencing of gene regions and analysis of allele-specific SNPs as described by Wolters et al. (2010). 
(a)

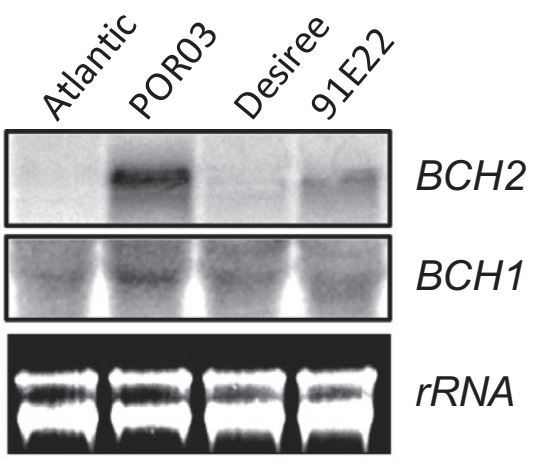

(b)

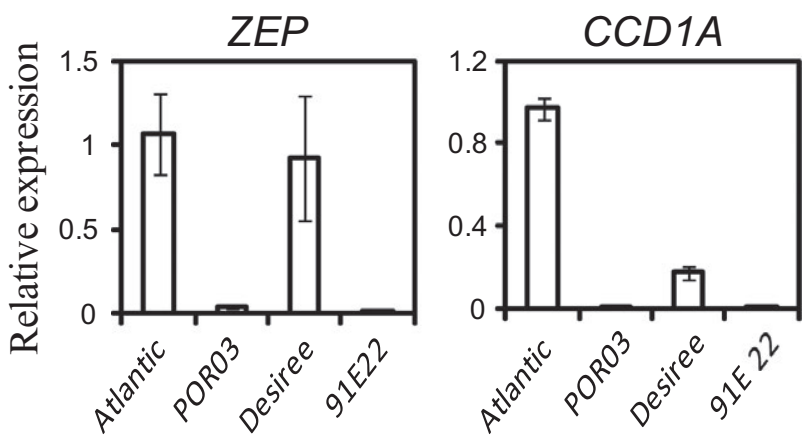

Figure 4. Transcript levels of differentially expressed carotenoid genes in tubers of four potato lines. (a) Northern blot analysis of $\mathrm{BCH} 2$ and $\mathrm{BCH} 1$ expression in tubers. Total RNA was extracted from pooled tubers and $20 \mu \mathrm{g}$ of RNA was resolved on a $1.2 \%$ denaturing gel. rRNA was used for equal loading control. (b) qRT-PCR analysis of ZEP and CCD1A expression. Total RNA $(5 \mu \mathrm{g})$ was used in reverse transcription. The experiments were repeated with three biological repeats.

Both diploid genotypes 91E22 and POR03 contained $B C H 2$ alleles 1 and 3 . Allele 3 is the dominant allele that has been shown to be associated with a high level of zeaxanthin accumulation in potato tuber (Wolters et al. 2010). Cultivar Désirée contained one $\mathrm{BCH} 2$ allele 3 together with one allele 1 and two alleles 2. Therefore, its $B C H 2$ allelic composition was 1,2,2,3. Cultivar Atlantic did not contain the dominant allele 3, but instead, one allele 1 and three alleles 5 (allelic composition 1,5,5,5). Alleles 1,2 and 5 are recessive alleles (Wolters et al. 2010).

Both diploid genotypes 91E22 and POR03 were homozygous for recessive $Z E P$ allele 1, which has a reduced level of expression in all diploid genotypes with orange tuber flesh (Wolters et al. 2010). The tetraploids Atlantic and Désirée did not contain $Z E P$ allele 1.

\section{Structural analysis of promoters of $\mathrm{BCH} 2$ genes}

$\mathrm{BCH} 2$ was expressed highly in the carotenoid-rich lines, indicating its important role in controlling carotenoid accumulation in tubers. To find potential cis-elements that control $\mathrm{BCH} 2$ expression in potato tubers, we isolated and compared the promoter sequences of different alleles of
$\mathrm{BCH} 2$ present in these four potato lines, which include the dominant allele 3 and the recessive alleles 1, 2 and 5. The $\mathrm{BCH} 2$ upstream promoter sequence of $1648 \mathrm{bp}$ (allele 5) and 1493 bp (allele 3) from Atlantic and POR03, respectively, was cloned through genome DNA walking. The $\mathrm{BCH} 2$ promoter sequences from allele 1 (1501 bp) and allele 2 (1713 bp) were obtained from monoploid genotypes 7322 and M5, respectively. Alignment of the four promoter sequences revealed 82 SNPs and 18 Indels, of which three were relatively large (Indel 7, 434 bp; Indel 10, 210 bp; Indel 14, 383 bp) (Supporting Information Fig. S1). Analysis of the regulatory regions of $B C H 2$ by PLACE (http://www. dna.affrc.go.jp/ PLACE/signalscan.html) (Higo et al. 1999) revealed several variable putative cis-element sequences (Supporting Information Fig. S1). Most noticeably, the promoter region of $\mathrm{BCH} 2$ from dominant allele 3 contains three unique cis-elements. One is recognized by MybSt1 (a potato MYB homolog), the second is recognized by AtMYC2 transcription factor and the third is WBOXHVISO1. WBOXHVISO1 has previously been reported to be involved in sugar signalling (Sun et al. 2003; Hammargren et al. 2008). That whether these motifs play a role in activating $\mathrm{BCH} 2$ expression of dominant allele 3 remains to be determined.

\section{Activity of the $\mathrm{BCH} 2$ promoters was stimulated by sugars}

WBOXHVISO1 box was found in Arabidopsis nucleoside diphosphate kinase (NDPK3a) and barley isol promoters and was shown to mediate sugar signalling and to be induced by sucrose and glucose (Sun et al. 2003; Hammargren et al. 2008). Potato tubers are starch-rich organs but also contain reducing sugars and sucrose. To examine the effect of sugars on induction of the $\mathrm{BCH} 2$ expression, the promoter sequences from recessive allele 5 (from Atlantic) and dominant allele 3 (from POR03) were fused to GUS reporter gene to produce PrO $_{\mathrm{BCH} 2-5}: G U S$ and $\mathrm{PrO}_{\mathrm{BCH}-3}: G U S$ constructs, respectively, and transformed into Arabidopsis. Transgenic Arabidopsis rosette leaves expressing ProBCH2:GUS constructs were treated with 6\% sucrose and glucose. No significant difference $(P>0.05)$ in GUS activity between PrO $_{\mathrm{BCH} 2-5}: G U S$ and $\mathrm{PrO}_{\mathrm{BCH} 2-3}: G U S$ was found in the detached rosette leaves treated with water (Fig. 5a). However, following treatment with $6 \%$ glucose or sucrose, an increase of GUS activity could be detected in transgenic leaves expressing both constructs. The GUS activity driven by $\mathrm{BCH} 2$ promoter from allele 3 in POR03 was significantly higher than that from allele 5 in Atlantic when the transgenic leaves were treated with glucose and sucrose. The results suggest that the higher expression of $\mathrm{BCH} 2$ in POR03 tubers may be associated with an enhanced response of the $\mathrm{BCH} 2$ promoter to sugars (Fig. 5a).

\section{Sugar levels in potato tubers}

In order to examine whether $\mathrm{BCH} 2$ expression was correlated with soluble sugars in potato tubers, the levels of reducing sugars of glucose, fructose and maltose and 
(a)

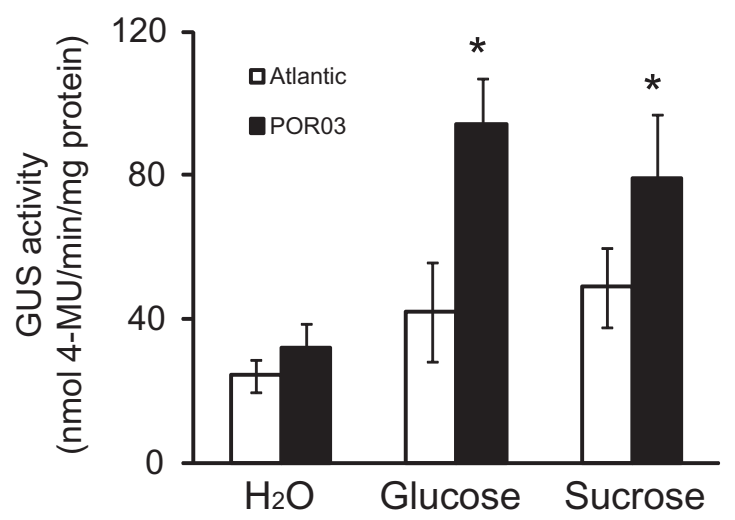

(b)
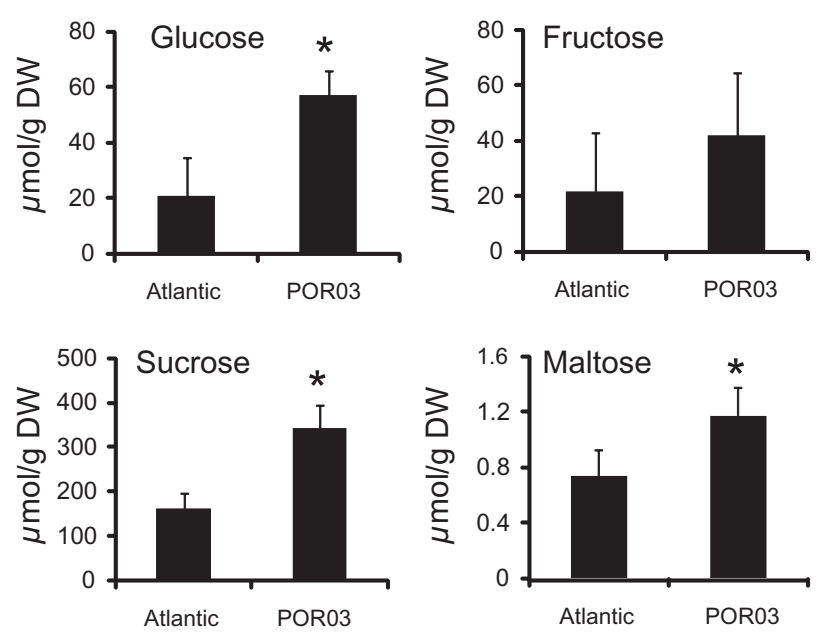

Figure 5. Analysis of promoter activity of $\mathrm{BCH} 2$ in transgenic Arabidopsis plants and sugar levels in potato lines Atlantic and POR03. (a) GUS activity driven by $\mathrm{BCH} 2$ promoters of allele 3 in POR03 and allele 5 in Atlantic after treatment with $6 \%$ sucrose and glucose for $24 \mathrm{~h}$. GUS activity was measured using 4-MUG as substrate. The analysis was repeated three times. (b) Sugar levels in potato tubers. Error bars indicate $+\mathrm{SD}$ of three repeat measurements. Values marked by asterisks indicate a statistically significant difference $(P<0.05)(n=3)$.

disaccharide sucrose were analysed in Atlantic and POR03. POR03 contained significantly $(P<0.05)$ more glucose than Atlantic (Fig. 5b). There was no significant difference in fructose level between Atlantic and POR03 $(P>0.05)$. Sucrose was the major soluble sugar detected in tubers (Fig. 5b). Sucrose content was doubled in POR3 compared with Atlantic. Maltose level in tubers was less than the other three soluble sugars, and POR3 contained significantly more maltose than Atlantic (Fig. 5b). Collectively, the results showed that the higher expression of $\mathrm{BCH} 2$ in POR03 than in Atlantic could result from its response to higher endogenous tuber glucose or sucrose levels.

\section{DISCUSSION}

Plants have evolved sophisticated mechanisms to control carbon flux into and through the carotenoid biosynthetic pathway. Evaluation of the accumulation of metabolites in the presence of pathway inhibitors, such as norflurazon, provides a convenient way to monitor carotenoid biosynthesis from overall carotenoid metabolism (Li et al. 2006). By measuring phytoene levels in potato tubers treated with norflurazon, we found that the increased carotenoid accumulation in potato tubers was associated with enhanced capacity of carotenoid biosynthesis. PSY as the first committed enzyme in carotenoid biosynthetic pathway is generally recognized to be the key in directing metabolic flux into the carotenoid biosynthetic pathway (Cazzonelli \& Pogson 2010). Alteration of its expression has profound effects on carotenoid accumulation in plants (Shewmaker et al.1999; Paine et al.2005). The upstream pathway enzyme 1-deoxy-D-xylulose 5-phosphate synthase (DXS) has also been shown to influence carotenoid biosynthesis (Enfissi et al. 2005). Previous studies in transgenic potato tubers show that alteration of some carotenoid gene expression plays an important role in carotenoid biosynthesis (Ducreux et al. 2005; Diretto et al. 2006). We compared the expression of PSY and DXS and found similar levels of expression among these four potato lines (data not shown), indicating that other unknown mechanisms likely regulate carotenoid metabolic flux in potato tubers.

Towards further understanding of the regulatory control of carotenoid accumulation in potato tubers, we conducted a genome-wide gene expression analysis between the white flesh cultivar Atlantic and the deep orange-yellow flesh line POR03. Genome-wide analysis identified four carotenoid metabolic pathway genes, i.e. $B C H 2, B C H 1, Z E P$ and $C C D 1 A$, which showed differential expression. $\mathrm{BCH} 2$ and $\mathrm{BCH} 1$ are non-heme di-iron enzymes. The biochemical function of $\mathrm{BCH}$ is to convert $\beta$-carotene to zeaxanthin. Two copies of $B C H$ are known in potato (Diretto et al. 2007), as well as in Arabidopsis (Sun, Gantt \& Cunningham 1996; Tian \& DellaPenna 2001) and tomato (Galpaz et al. 2006). Here, we showed that $B C H 2$ was the most highly differentially expressed gene and its transcript levels were tightly associated with carotenoid content in these four potato lines, consistent with previous studies obtained by association mapping and a microarray analysis (Brown et al. 2006; Kloosterman et al. 2010; Wolters et al. 2010). The results suggest that $\mathrm{BCH} 2$ functions as a major factor in directing the flux through the $\beta$-branch of the pathway to lead zeaxanthin synthesis in tubers. Unlike tomato $B C H 1$, which is believed to be mainly involved in carotenoid biosynthesis in photosynthesis tissue (Galpaz et al. 2006), a correlated expression of $\mathrm{BCH} 1$ is also observed among potato cultivars, indicating that it could play a role, even if less important than $\mathrm{BCH}$, in zeaxanthin biosynthesis in tubers.

ZEP is responsible for the conversion of zeaxanthin to violaxanthin. We found that $Z E P$ transcript level was drastically reduced in POR03 and 91E22 compared with Atlantic and Désirée. Reduction of $Z E P$ expression is reported to cause to a dramatic increase of zeaxanthin content, as well as the total tuber carotenoid level in transgenic potato lines (Romer et al. 2002). Similarly, low transcript levels are 
observed in high carotenoid lines from examination of a range of potato germplasm (Morris et al. 2004). It is apparent that tuber-specific expression of $Z E P$ adversely affects tuber carotenoid content.

Zeaxanthin was the major component of carotenoids accumulated in tubers of the diploid genotypes POR03 and 91E22. The enhanced expression of $\mathrm{BCH} 2$ and the reduced expression of $Z E P$ were consistent with the recent report showing that the presence of a dominant $\mathrm{BCH} 2$ allele 3 and a homozygous recessive $Z E P$ allele 1 is responsible for zeaxanthin accumulation in orange-yellow flesh tubers (Wolters et al. 2010). Eight BCH2 alleles have been identified from potato and the presence of a single allele 3 is sufficient for its yellow flesh colour (Wolters et al.2010). We examined the $\mathrm{BCH} 2$ and $\mathrm{ZEP}$ allele compositions of the four potato lines. While the $\mathrm{BCH} 2$ allele composition for white flesh cultivar Atlantic was 1,5,5,5, Désirée had alleles $1,2,2,3$, and the diploid lines contained alleles 1 and 3 . Although one allele 3 was present in POR03, 91E22 and Désirée, the different levels of carotenoid accumulation among them confirm the role of additional genes in controlling total carotenoid accumulation in potato tubers as suggested (Brown et al. 2006). Five ZEP alleles have been identified in potato and a recessive homozygous allele 1 is found to be associated with orange-yellow flesh phenotype (Wolters et al. 2010). The high carotenoid lines POR03 and 91E22 were homozygous for ZEP allele 1 with low transcript level. The low expression reduces the rate for further metabolizing zeaxanthin to other xanthophylls. In contrast, Désirée did not contain allele 1 and showed much higher transcription of ZEP than POR03 and 91E22, which offers an explanation for lower-level carotenoid accumulation in Désirée in comparison with the high carotenoid lines.

A family of CCDs catalyses enzymatic turnover of carotenoids for the production of apocarotenoids, such as $\beta$-ionone, pseudoionone and geranylacetone (Auldridge, McCarty \& Klee 2006). In Arabidopsis, there are nine members of CCDs that cleave multiple carotenoid substrates into apocarotenoids (Tan et al. 2003). CCDs help maintain the homeostasis of carotenoids in plants (Ohmiya et al.2006). CCD1A, a homolog of tomato CCD1A (Simkin et al. 2004), was identified here to be differentially expressed between Atlantic and POR03, and its transcript level was found to be dramatically reduced in high carotenoid lines. A dramatic reduced expression of $C C D 1 A$ in high carotenoid varieties suggests a reduced carotenoid turnover. A recent study of another member of the $C C D$ gene family, $C C D 4$, in potato shows that down-regulation of $C C D 4$ by RNAi results in two- to fivefold increased carotenoids in potato tubers (Campbell et al. 2010). It appears that CCD activity affects final carotenoid accumulation in potato tubers, but the role of individual member varies depending on varieties.

Sugars mainly sucrose have been shown to induce expression of some tuber-specific genes, such as patatin, sucrose synthase Sus4-16 and StCDPK1 in other tissues where they are not normally expressed (Grierson et al. 1994; Fu, Kim \& Park 1995; Raices et al. 2003). Sucrose promotes conversion of chloroplasts into chromoplasts for carotenoid accumulation in citrus peel segments (Huff 1983). Sucrose availability affects PSY1 expression and lycopene accumulation in tomato fruits (Télef et al. 2006). A number of carotenoid biosynthetic genes are transcriptionally activated by glucose in cyanobacterium Synechocystis (Ryu et al. 2004). Here, we found that $\mathrm{BCH} 2$ expression was activated in leaf tissue by exogenous application of glucose and sucrose, indicating that free sugars in tubers could play a role in promoting $\mathrm{BCH} 2$ expression. Indeed, POR03 tubers contained more glucose and sucrose than Atlantic. This provides an explanation for high expression of $\mathrm{BCH} 2$ in POR03 tubers. Further analysis of the sugar-responsive ciselement in the $\mathrm{BCH} 2$ promoter will help dissect the local network regulating carotenogenesis in potato tubers.

Taken together, our results showed that the high carotenoid accumulation in potato tubers is due to a combination of increased metabolic flux into the carotenoid biosynthetic pathway and the transcriptional control of a number of carotenoid metabolic genes. The synergistic effect of elevated expression of $\mathrm{BCH} 2$ and $\mathrm{BCH} 1$ as well as suppressed expression of $Z E P$ and $C C D 1 A$ is responsible for zeaxanthin accumulation in the yellow-fleshed varieties.

\section{ACKNOWLEDGMENTS}

We thank the Plant Cell Imaging Center at Boyce Thompson Institute for Plant Research for sharing the facilities, which were sponsored by NSF (NSF DBI-0618969) and The TRIAD Foundation. This research was supported in part by USDA National Research Initiative Competitive Grant 2007-35318-17794.

\section{REFERENCES}

Auldridge M.E., McCarty D.R. \& Klee H.J. (2006) Plant carotenoid cleavage oxygenases and their apocarotenoid products. Current Opinion in Plant Biology 9, 315-321.

Benjamini Y. \& Hochberg Y. (1995) Controlling the false discovery rate: a practical and powerful approach to multiple testing. Journal of the Royal Statistical Society 57, 289-300.

Bonierbale M.W., Plaisted R.L. \& Tanksley S.D. (1988) RFLP maps based on a common set of clones reveal modes of chromosomal evolution in potato and tomato. Genetics 120, 1095-1103.

Breitenbach J., Zhu C. \& Sandmann G. (2001) Bleaching herbicide norflurazon inhibits phytoene desaturase by competition with the cofactors. Journal of Agricultural and Food Chemistry 49, 5270-5272.

Brown C.R., Kim T.S., Ganga Z., Haynes K., De Jong D., Jahn M., Paran I. \& De Jong W. (2006) Segregation of total carotenoid in high level potato germplasm and its relationship to beta-carotene hydroxylase polymorphism. American Journal of Potato Research 83, 365-372.

Brown C., Durst R., Wrolstad R. \& De Jong W. (2008) Variability of phytonutrient content of potato in relation to growing location and cooking method. Potato Research 51, 259-270.

Burgos G., Salas E., Amoros W., Auqui M., Muñoa L., Kimura M. \& Bonierbale M. (2009) Total and individual carotenoid profiles in Solanum phureja of cultivated potatoes: I. Concentrations and 
relationships as determined by spectrophotometry and HPLC. Journal of Food Composition and Analysis 22, 503-508.

Campbell R., Ducreux L.J., Morris W.L., Morris J.A., Suttle J.C., Ramsay G., Bryan G.J., Hedley P.E. \& Taylor M.A. (2010) The metabolic and developmental roles of carotenoid cleavage dioxygenase4 from potato. Plant Physiology 154, 656-664.

Cazzonelli C.I. \& Pogson B.J. (2010) Source to sink: regulation of carotenoid biosynthesis in plants. Trends in Plant Science 15, 266-274.

Chiu L.W., Zhou X., Burke S., Wu X., Prior R.L. \& Li L. (2010) The purple cauliflower arises from activation of a MYB transcription factor. Plant Physiology 154, 1470-1480.

Davuluri G.R., van Tuinen A., Fraser P.D., et al. (2005) Fruitspecific RNAi-mediated suppression of DET1 enhances carotenoid and flavonoid content in tomatoes. Nature Biotechnology 23, 890-895.

Diretto G., Tavazza R., Welsch R., Pizzichini D., Mourgues F., Papacchioli V., Beyer P. \& Giuliano G. (2006) Metabolic engineering of potato tuber carotenoids through tuber-specific silencing of lycopene epsilon cyclase. BMC Plant Biology 6, 13 .

Diretto G., Al-Babili S., Tavazza R., Papacchioli V., Beyer P. \& Giuliano G. (2007) Metabolic engineering of potato carotenoid content through tuber-specific overexpression of a bacterial mini-pathway. PLOS ONE 2, e350.

Diretto G., Al-Babili S., Tavazza R., Scossa F., Papacchioli V., Migliore M., Beyer P. \& Giuliano G. (2010) Transcriptionalmetabolic networks in $\beta$-carotene-enriched potato tubers: the long and winding road to the Golden phenotype. Plant Physiology 154, 899-912.

Ducreux L.J.M., Morris W.L., Hedley P.E., Shepherd T., Davies H.V., Millam S. \& Taylor M.A. (2005) Metabolic engineering of high carotenoid potato tubers containing enhanced levels of beta-carotene and lutein. Journal of Experimental Botany 56, 81-89.

Enfissi E.M.A., Fraser P.D., Lois L.M., Boronat A., Schuch W. \& Bramley P.M. (2005) Metabolic engineering of the mevalonate and non-mevalonate isopentenyl diphosphate-forming pathways for the production of health-promoting isoprenoids in tomato. Plant Biotechnology Journal 3, 17-27.

Fei Z., Joung J.G., Tang X., et al. (2011) Tomato functional genomics database: a comprehensive resource and analysis package for tomato functional genomics. Nucleic Acids Research 39, D1156D1163.

Fraser P.D. \& Bramley P.M. (2004) The biosynthesis and nutritional uses of carotenoids. Progress in Lipid Research 43, 228-265.

Fraser P.D., Romer S., Shipton C.A., Mills P.B., Kiano J.W., Misawa N., Drake R.G., Schuch W. \& Bramley P.M. (2002) Evaluation of transgenic tomato plants expressing an additional phytoene synthase in a fruit-specific manner. Proceedings of the National Academy of Sciences of the United States of America 99, 10921097.

Fu H., Kim S.Y. \& Park W.D. (1995) High-level tuber expression and sucrose inducibility of a potato Sus4 sucrose synthase gene require $5^{\prime}$ and $3^{\prime}$ flanking sequences and the leader intron. The Plant Cell 7, 1387-1394.

Galpaz N., Ronen G., Khalfa Z., Zamir D. \& Hirschberg J. (2006) A chromoplast-specific carotenoid biosynthesis pathway is revealed by cloning of the tomato white-flower locus. The Plant Cell 18, 1947-1960.

Galpaz N., Wang Q., Menda N., Zamir D. \& Hirschberg J. (2008) Abscisic acid deficiency in the tomato mutant high-pigment 3 leading to increased plastid number and higher fruit lycopene content. The Plant Journal 53, 717-730.

Giuliano G., Tavazza R., Diretto G., Beyer P. \& Taylor M.A. (2008) Metabolic engineering of carotenoid biosynthesis in plants. Trends in Biotechnology 26, 139-145.
Grant G.R., Liu J. \& Stoeckert C.J., Jr (2005) A practical false discovery rate approach to identifying patterns of differential expression in microarray data. Bioinformatics 21, 2684-2690.

Grierson C., Du J.S., de Torres Z.M., Beggs K., Smith C., Holdsworth M. \& Bevan M. (1994) Separate cis sequences and trans factors direct metabolic and developmental regulation of a potato tuber storage protein gene. The Plant Journal 5, 815-826.

Griffiths A., Barry C., Puche-Solis A. \& Grierson D. (1999) Ethylene and developmental signals regulate expression of lipoxygenase genes during tomato fruit ripening. Journal of Experimental Botany 50, 793-798.

Hammargren J., Rosenquist S., Jansson C. \& Knorpp C. (2008) A novel connection between nucleotide and carbohydrate metabolism in mitochondria: sugar regulation of the Arabidopsis nucleoside diphosphate kinase 3a gene. Plant Cell Reports 27, 529-534.

Harjes C.E., Rocheford T.R., Bai L., et al. (2008) Natural genetic variation in lycopene epsilon cyclase tapped for maize biofortification. Science 319, 330-333.

Higo K., Ugawa Y., Iwamoto M. \& Korenaga T. (1999) Plant cisacting regulatory DNA elements (PLACE) database: 1999. Nucleic Acids Research 27, 297-300.

Huff A. (1983) Nutritional control of regreening and degreening in citrus peel segments. Plant Physiology 73, 243-249.

Joung J.G., Corbett A.M., Fellman S.M., Tieman D.M., Klee H.J., Giovannoni J.J. \& Fei Z. (2009) Plant MetGenMAP: an integrative analysis system for plant systems biology. Plant Physiology 151, 1758-1768.

Kaplan F. \& Guy C.L. (2004) beta-Amylase induction and the protective role of maltose during temperature shock. Plant Physiology 135, 1674-1684.

Kato M., Matsumoto H., Ikoma Y., Okuda H. \& Yano M. (2006) The role of carotenoid cleavage dioxygenases in the regulation of carotenoid profiles during maturation in citrus fruit. Journal of Experimental Botany 57, 2153-2164.

Kloosterman B., Oortwijn M., uitdeWilligen J., America T., de Vos R., Visser R.G.F. \& Bachem C.W.B. (2010) From QTL to candidate gene: genetical genomics of simple and complex traits in potato using a pooling strategy. BMC Genomics 11, 158.

Kobayashi A., Ohara-Takada A., Tsuda S., Matsuura-Endo C., Takada N., Umemura Y., Nakao T., Yoshida T., Hayashi K. \& Mori M. (2008) Breeding of potato variety 'Inca-no-hitomi' with a very high carotenoid content. Breeding Science 58, 77-82.

Li L. \& Van Eck J. (2007) Metabolic engineering of carotenoid accumulation by creating a metabolic sink. Transgenic Research 16, 581-585.

Li L., Paolillo D.J., Parthasarathy M.V., Dimuzio E.M. \& Garvin D.F. (2001) A novel gene mutation that confers abnormal patterns of beta-carotene accumulation in cauliflower (Brassica oleracea var. botrytis). The Plant Journal 26, 59-67.

Li L., Lu S., Cosman K.M., Earle E.D., Garvin D.F. \& O'Neill J. (2006) Beta-carotene accumulation induced by the cauliflower Or gene is not due to an increased capacity of biosynthesis. Phytochemistry 67, 1177-1184.

Liu Y., Roof S., Ye Z., Barry C., van Tuinen A., Vrebalov J., Bowler C. \& Giovannoni J. (2004) Manipulation of light signal transduction as a means of modifying fruit nutritional quality in tomato. Proceedings of the National Academy of Sciences of the United States of America 101, 9897-9902.

Lopez A.B., Van E.J., Conlin B.J., Paolillo D.J., O’Neill J. \& Li L. (2008) Effect of the cauliflower Or transgene on carotenoid accumulation and chromoplast formation in transgenic potato tubers. Journal of Experimental Botany 59, 213-223.

Lu S. \& Li L. (2008) Carotenoid metabolism: biosynthesis, regulation, and beyond. Journal of Integrative Plant Biology 50, 778785 . 
Lu S., Van Eck J., Zhou X., et al. (2006) The cauliflower Or gene encodes a DnaJ cysteine-rich domain-containing protein that mediates high levels of beta-carotene accumulation. The Plant Cell 18, 3594-3605.

Moore S., Payton P., Wright M., Tanksley S. \& Giovannoni J. (2005) Utilization of tomato microarrays for comparative gene expression analysis in the Solanaceae. Journal of Experimental Botany 56, 2885-2895.

Morris W.L., Ducreux L., Griffiths D.W., Stewart D., Davies H.V. \& Taylor M.A. (2004) Carotenogenesis during tuber development and storage in potato. Journal of Experimental Botany 55, 975982.

Naqvi S., Farre G., Sanahuja G., Capell T., Zhu C. \& Christou P. (2010) When more is better: multigene engineering in plants. Trends in Plant Science 15, 48-56.

Ohmiya A., Kishimoto S., Aida R., Yoshioka S. \& Sumitomo K. (2006) Carotenoid cleavage dioxygenase (CmCCD4a) contributes to white color formation in chrysanthemum petals. Plant Physiology 142, 1193-1201.

Paine J.A., Shipton C.A., Chaggar S., et al. (2005) Improving the nutritional value of Golden Rice through increased pro-vitamin A content. Nature Biotechnology 23, 482-487.

Ponce-Valadez M., Fellman S.M., Giovannoni J., Gan S.S. \& Watkins C.B. (2009) Differential fruit gene expression in two strawberry cultivars in response to elevated $\mathrm{CO}_{2}$ during storage revealed by a heterologous fruit microarray approach. Postharvest Biology and Technology 51, 131-140.

Raices M., Ulloa R.M., MacIntosh G.C., Crespi M. \& Tellez-Inon M.T. (2003) StCDPK1 is expressed in potato stolon tips and is induced by high sucrose concentration. Journal of Experimental Botany 54, 2589-2591.

Romer S., Lubeck J., Kauder F., Steiger S., Adomat C. \& Sandmann G. (2002) Genetic engineering of a zeaxanthin-rich potato by antisense inactivation and co-suppression of carotenoid epoxidation. Metabolic Engineering 4, 263-272.

Ronen G., Cohen M., Zamir D. \& Hirschberg J. (1999) Regulation of carotenoid biosynthesis during tomato fruit development: expression of the gene for lycopene epsilon-cyclase is downregulated during ripening and is elevated in the mutant Delta. The Plant Journal 17, 341-351.

Ryu J.Y., Song J.Y., Lee J.M., Jeong S.W., Chow W.S., Choi S.B., Pogson B.J. \& Park Y.I. (2004) Glucose-induced expression of carotenoid biosynthesis genes in the dark is mediated by cytosolic $\mathrm{pH}$ in the cyanobacterium Synechocystis sp. PCC 6803. Journal of Biological Chemistry 279, 25320-25325.

Shewmaker C.K., Sheehy J.A., Daley M., Colburn S. \& Ke D.Y. (1999) Seed-specific overexpression of phytoene synthase: increase in carotenoids and other metabolic effects. The Plant Journal 20, 401-412.

Simkin A.J., Schwartz S.H., Auldridge M., Taylor M.G. \& Klee H.J. (2004) The tomato carotenoid cleavage dioxygenase 1 genes contribute to the formation of the flavor volatiles beta-ionone, pseudoionone, and geranylacetone. The Plant Journal 40, 882892.

Stupar R.M., Bhaskar P.B., Yandell B.S., et al. (2007) Phenotypic and transcriptomic changes associated with potato autopolyploidization. Genetics 176, 2055-2067.

Sun Z., Gantt E. \& Cunningham F.X. (1996) Cloning and functional analysis of the beta-carotene hydroxylase of Arabidopsis thaliana. Journal of Biological Chemistry 271, 24349-24352.

Sun C., Palmqvist S., Olsson H., Boren M., Ahlandsberg S. \& Jansson C. (2003) A novel WRKY transcription factor, SUSIBA2, participates in sugar signaling in barley by binding to the sugar-responsive elements of the iso1 promoter. The Plant Cell 15, 2076-2092.

Tan B.C., Joseph L.M., Deng W.T., Liu L., Li Q.B., Cline K. \& McCarty D.R. (2003) Molecular characterization of the Arabidopsis 9-cis epoxycarotenoid dioxygenase gene family. The Plant Journal 35, 44-56.

Taylor M. \& Ramsay G. (2005) Carotenoid biosynthesis in plant storage organs: recent advances and prospects for improving plant food quality. Physiologia Plantarum 124, 143-151.

Télef N., Stammitti-Bert L., Mortain-Bertrand A., Maucourt M., Carde J., Rolin D. \& Gallusci P. (2006) Sucrose deficiency delays lycopene accumulation in tomato fruit pericarp discs. Plant Molecular Biology 62, 453-469.

Tian L. \& DellaPenna D. (2001) Characterization of a second carotenoid beta-hydroxylase gene from Arabidopsis and its relationship to the LUT1 locus. Plant Molecular Biology 47, 379-388.

Van Eck J., Conlin B., Garvin D.F., Mason H., Navarre D.A. \& Brown C.R. (2007) Enhancing beta-carotene content in potato by RNAi-mediated silencing of the beta-carotene hydroxylase gene. American Journal of Potato Research 84, 331-342.

Wolters A.M.A., Uitdewilligen J.G.A.M.L., Kloosterman B.A., Hutten R.C.B., Visser R.G.F. \& van Eck H.J. (2010) Identification of alleles of carotenoid pathway genes important for zeaxanthin accumulation in potato tubers. Plant Molecular Biology 73, 659-671.

Yan J., Kandianis C.B., Harjes C.E., et al. (2010) Rare genetic variation at Zea mays crtRB1 increases $\beta$-carotene in maize grain. Nature Genetics 42, 322-327.

Yang Y.H., Dudoit S., Luu P., Lin D.M., Peng V., Ngai J. \& Speed T.P. (2002) Normalization for cDNA microarray data: a robust composite method addressing single and multiple slide systematic variation. Nucleic Acids Research 30, e15.

Zhou X., Cooke P. \& Li L. (2010) Eukaryotic release factor 1-2 affects Arabidopsis responses to glucose and phytohormones during germination and early seedling development. Journal of Experimental Botany 61, 357-367.

Received 21 December 2010; received in revised form 18 January 2011; accepted for publication 30 January 2011

\section{SUPPORTING INFORMATION}

Additional Supporting Information may be found in the online version of this article:

Figure S1. Structure analysis of the $B C H 2$ promoters from Atlantic, Désirée, 91E22, and POR03.

Table S1. Primers used for amplification of probes for northern blot analysis, genome walking, and qRT-PCR analysis of genes differentially expressed based on microarray data.

Table S2. Top 100 up-regulated genes in microarray analysis of potato tubers from Altantic and POR03.

Table S3. Top 100 down-regulated genes in microarray analysis of potato tubers from Altantic and POR03.

Please note: Wiley-Blackwell are not responsible for the content or functionality of any supporting materials supplied by the authors. Any queries (other than missing material) should be directed to the corresponding author for the article. 